\title{
Thoracoscopic pulmonary vein isolation in patients with atrial fibrillation and failed percutaneous ablation
}

\author{
Manuel Castellá, MD, PhD, Daniel Pereda, MD, Carlos A. Mestres, MD, PhD, FETCS, Félix Gómez, MD, \\ Eduard Quintana, MD, and Jaume Mulet, MD, PhD
}

Objective: Pulmonary vein isolation is indicated in patients with symptomatic isolated atrial fibrillation not controlled with antiarrhythmic therapy. We describe our surgical experience with thoracoscopic pulmonary vein isolation in patients in whom percutaneous ablation has failed.

\begin{abstract}
Methods: Thirty-four adult patients with unsuccessful catheter ablations (range 1-4, mean $2 \pm 1$ ) underwent thoracoscopic bipolar-radiofrequency pulmonary vein isolation. Seventeen patients had paroxysmal atrial fibrillation, 12 with persistent and 5 with long-standing persistent fibrillation, for a mean of 6 years (range 3-10 years), 13 years (5-25 years), and 9 years (3-15 years), respectively.

Results: There was no mortality during the procedure or follow-up (mean $16 \pm 11$ months). Two patients needed conversion to thoracotomy owing to hemorrhage, and ablation could not be completed. Antiarrhythmic therapy was withdrawn 3 months postoperatively. Postoperative sinus rhythm was maintained in $82 \%$ of those with paroxysmal atrial fibrillation (13/15 at 6 months, $9 / 11$ at 12 months), $60 \%$ had persistent atrial fibrillation $(8 / 12$ at 6 months and 6/10 at 12 months), and 20\% had long-standing persistent atrial fibrillation (1/5 at 6 and 12 months). Preoperative left atrial diameter significantly differed between patients with paroxysmal fibrillation (mean $42 \pm 6$ $\mathrm{mm}$ ) and those with persistent and long-standing persistent fibrillation (means $50 \pm 4$ and $47 \pm 2 \mathrm{~mm}$ ). Left atrial size greater than $45 \mathrm{~mm}$ and atrial fibrillation type were preoperative factors that significantly influenced outcome in the univariate logistic regression analysis.
\end{abstract}

Conclusions: Thoracoscopic pulmonary vein isolation in patients with previously unsuccessful catheter ablations demonstrates satisfactory sinus rhythm maintenance rates in paroxysmal and persistent atrial fibrillation, but not in long-standing persistent atrial fibrillation. As with other minimally invasive surgical techniques, there is an important learning curve. (J Thorac Cardiovasc Surg 2010;140:633-8)

In the latest consensus guidelines on atrial fibrillation (AF) therapy by the American College of Cardiology (ACC), the American Heart Association (AHA), and the European Society of Cardiology (ESC), published in 2006, catheterbased pulmonary vein isolation is a good treatment choice in patients with paroxysmal symptomatic AF not controlled with antiarrhythmic drugs or in patients with recurrent persistent AF if they remain symptomatic with rate control therapy. ${ }^{1}$ These recommendations were based on the knowledge that most of the trigger zones that originate AF can be found in the left atrium around the pulmonary veins, ${ }^{2}$ and by the introduction of percutaneous catheter technology allowing

\footnotetext{
From the Department of Cardiovascular Surgery, Institut del Tòrax, Hospital Clínic, University of Barcelona, Barcelona, Spain.

Disclosures: The authors had full control of the design of the study, methods used, outcome parameters, analysis of data, and production of the written report. Manuel Castellá reports consulting fees and grant support from Atricure, Inc.

Received for publication July 9, 2009; revisions received Oct 7, 2009; accepted for publication Nov 4, 2009; available ahead of print Feb 1, 2010.

Address for reprints: Manuel Castellá, MD, PhD, Department of Cardiovascular Surgery, Hospital Clínic, Villarroel 170,08036-Barcelona, Spain (E-mail: mcaste@ clinic.ub.es).

$0022-5223 / \$ 36.00$

Copyright (c) 2010 by The American Association for Thoracic Surgery

doi:10.1016/j.jtcvs.2009.11.009
}

radiofrequency isolation of the left atrium at the antrum of the pulmonary veins. In 2007, the Heart Rhythm Society (HRS), European Heart Rhythm Association (EHRA), and European Cardiac Arrhythmia Society (ECAS) Task Force on Catheter and Surgical Ablation of AF developed an Expert Consensus Statement extending ablation indications to all symptomatic AF refractory or intolerant to antiarrhythmic medication and, in rare clinical situations, as first-line therapy. ${ }^{3}$ It is important to mention that these guideline algorithms focus only on catheter ablation when referring to isolated $\mathrm{AF}$ treatment, with no mention of surgical ablation strategies. Nevertheless, percutaneous ablation is curative in around $70 \%$ to $80 \%$ of patients with paroxysmal $\mathrm{AF}$ and normal left atrial size. The incidence of major complications remains at $5 \%{ }^{4}$ Success rates drop in patients with persistent $\mathrm{AF}$, long-standing persistent $\mathrm{AF}$, or an enlarged left atrium. There is much speculation why catheter ablation is not fully successful. First, there is the technologic issue of achieving complete isolation. Gaps created when performing a line of endocardial ablation dots likely affect success rates. This effect is increased in enlarged atria, where lines need to be longer. Often, to avoid atrial perforation and transesophageal fistulas, the amount of energy applied during the procedure must be limited. In addition, the left atrial 


\section{Abbreviations and Acronyms \\ $\mathrm{AF}=$ atrial fibrillation \\ $\mathrm{AUC}=$ area under the curve \\ $\mathrm{CI}=$ confidence interval \\ $\mathrm{IVC}=$ inferior vena cava}

wall thickness can vary from patient to patient. All of these factors can affect success rates. It is therefore possible that lines can be incomplete when drawn during a percutaneous procedure in which a closed approach is used and visualization is limited. In addition, myocardial remodeling and fibrosis that can occur in patients with an enlarged atrium and persistent or long-standing persistent AF may necessitate more than isolation of possible trigger zones around pulmonary veins. These types of patients need to be more focused toward a true maze approach owing to myocardial remodeling and fibrosis.

The possibility of curing paroxysmal AF by pulmonary vein isolation combined with new energy sources has led to newer types of surgical strategies focused on minimally invasive procedures. ${ }^{5}$ Current surgical devices are able to deliver radiofrequency energy via gold-plated electrodes placed around the pulmonary veins. These clamps produce a consistent line of ablation. Most important, transmurality can be confirmed by a fall in electrical conductance along the tissue ablated.

This study reports our experience with symptomatic patients with AF in whom repetitive percutaneous pulmonary vein ablation has failed. Our purpose was to surgically ensure transmural ablation lines in these patients, to confirm that percutaneous catheter ablation failures were due to defects in pulmonary vein isolation and to evaluate whether persistent and long-standing persistent $\mathrm{AF}$ are influenced by pulmonary vein isolation alone.

\section{PATIENTS AND METHODS}

From May 2005 to September 2008, 34 patients were selected for thoracoscopically assisted pulmonary vein isolation according to the following criteria: symptomatic AF that did not respond to medical therapy and failure to eradicate the arrhythmia by percutaneous radiofrequency ablation of the left atrium. All patients had one or more of the following symptoms before surgery: palpitations, shortness of breath at high cardiac rates, and anxiety for the possibility of a symptomatic AF episode. Patterns of percutaneous monopolar radiofrequency lesions in the preoperative catheter ablations included pulmonary vein ablation in all patients. The ablation lines ran between the left and right pulmonary veins, between the left inferior pulmonary vein and the mitral annulus, and between the inferior vena cava (IVC) and the tricuspid annulus in most patients (preoperative data in Table 1). Percutaneous ablations were performed a mean of 2 times, with a range from 1 (10 patients) to 4 times (1 patient). Entrance and exit blocks were confirmed after each percutaneous ablation and were required to be considered successful in all procedures and all patients. Nevertheless, all patients continued to have their AF pattern for at least 3 months after the percutaneous ablation, except 3 patients in whom the endoluminal treatment
TABLE 1. Preoperative characteristics of the 32 patients with complete ablation procedure

\begin{tabular}{lcccc}
\hline & Mean & Median & SD & Range \\
\hline Age (y) & 54 & 56 & 8.62 & $28-66$ \\
AF duration (y) & 8.10 & 7.00 & 4.84 & $2-25$ \\
Number of previous & 2.06 & 2.00 & 0.91 & $1-4$ \\
$\quad \begin{array}{l}\text { percutaneous procedures } \\
\text { Left atrial size (mm) }\end{array}$ & 45.34 & 45.50 & 5.61 & $32-54$ \\
\hline SD, Standard deviation; $A F$, atrial fibrillation. & & &
\end{tabular}

transformed their pattern from persistent to paroxysmal. Three patients had perforation of the left atrium during percutaneous ablation, and 1 patient with persistent AF needed emergency sternotomy to control bleeding. The patient was treated with surgical ablation during that sternotomy. This was the only patient in whom the surgical access was not thoracoscopic. In another patient with a history of atrial bleeding during percutaneous ablation necessitating emergency subxiphoid drainage, important pericardial adhesions were detected at surgery 6 months later. A 28 -yearold patient had asymptomatic severe stenoses of the left pulmonary veins from the first ablation performed with the lasso technique 6 years before the operation. ${ }^{6}$ These stenoses produced a hemodynamic gradient of 16 $\mathrm{mm} \mathrm{Hg}(2 \mathrm{~m} / \mathrm{s})$ through the narrowest points of the superior and inferior veins at the entrance to the left atrium, 4.8 and $5.8 \mathrm{~mm}$ in diameter, respectively, compared with 19.8 and $18.7 \mathrm{~mm}$ in the right side. Last, another patient had Fontaine grade IIa inferior limb ischemia owing to a femoral arteriovenous fistula after puncture for percutaneous ablation that needed reconstructive vascular surgery 1 week after thoracoscopic pulmonary vein isolation.

All patients had isolated AF, without any history of coronary artery or valvular disease. Sixteen $(47 \%)$ of 34 patients were treated for arterial hypertension, which was well controlled in all of them. Preoperative echocardiogram showed normal function of the left and right ventricles in all patients except for one with severe left ventricular dysfunction attributed to tachycardiomyopathy (left ventricular ejection fraction 35\%, no segmental contraction deficits, and repetitive negative stress tests). No valvular dysfunctions were detected, nor were there any thrombi in the left atrial appendage. Left atrial diameter was significantly smaller in the patients with paroxysmal $\mathrm{AF}$ as opposed to those with persistent and long-standing persistent $\mathrm{AF}$ ( $42 \pm 6 \mathrm{~mm}$ vs $50 \pm 4 \mathrm{~mm}$, respectively; $P<.02)$. Although time from AF initiation did not significantly differ between the types of AF patterns, patients with paroxysmal $\mathrm{AF}$ had less time from first episodes until surgery (Table 1). There were no differences in age or prevalence of arterial hypertension, known factors favoring $\mathrm{AF}^{7}$

\section{Procedural Technique}

All patients except one were operated on through video-assisted thoracoscopic surgery. The one exception, with persistent AF, needed emergency surgery after left atrial perforation and cardiac tamponade in the electrophysiology laboratory, so the procedure was performed through a sternotomy after the left atrium had been sutured.

In the video-assisted surgery, the procedure was conducted after general anesthesia and double-lumen endotracheal intubation. The patient was placed in the left lateral decubitus position to access the right pulmonary veins through two 5-mm ports in the sixth intercostal space and a 50-mm working port in the third, with the use of a soft tissue retractor (Edwards Lifesciences CardioVations, Irvine, Calif), as described previously. ${ }^{5}$ After lung deflation, the pericardium was divided $2 \mathrm{~cm}$ anterior to the phrenic nerve from the superior vena cava to the IVC, with the visual help of a $30^{\circ}, 5-\mathrm{mm}$ thoracoscope. Blunt dissection was used to access the oblique sinus between the right inferior pulmonary vein and the IVC, and both right pulmonary veins were surrounded with the help of an articulated lighted 
dissector (AtriCure, Inc, Cincinnati, Ohio). A Red Rubber catheter was placed around the pulmonary veins. A 5-cm long bipolar radiofrequency clamp (AtriCure) was advanced with the rubber catheter attached to the tip of the lower jaw to guide it through the oblique sinus, until its tip could be visualized cephalic to the superior pulmonary vein. Bipolar ablation was accomplished by closing the bipolar clamp along a left atrial cuff adjacent to the entrance of the pulmonary veins, ensuring not to ablate the veins. The clamp produces a linear thermal lesion by radiofrequency energy, and transmurality was accomplished when tissue conductance dropped under 0.0025 siemens, normally after 8 to 15 seconds of energy delivery. After testing for transmurality as specified below, the lung was reinflated, a chest tube was inserted through one of the small ports, and the other thoracic accesses were closed. The patient was then placed in the right lateral decubitus position to access the left pulmonary veins and the left atrial appendage.

Placement of thoracoscopic ports on the left side was similar to the right. After division of the ligament of Marshall, both left pulmonary veins were surrounded by a Red Rubber catheter with the help of the articulated lighted dissector. The rubber catheter was used to guide the radiofrequency clamp to be able to isolate a left atrial cuff surrounding the entrance of the pulmonary veins. Once isolation was completed, the left atrial appendage was stapled and excised with an automatic suture (ETS-Flex 45;Ethicon Endosurgery Inc, Cincinnati, Ohio).

Ablation lesions are repeated 3 to 5 times on each side to widen the isolation line. Before and after ablation, measurements of isolation were performed by pacing within the pulmonary veins (exit block) and with ganglionic plexi stimulation. Patients in AF were cardioverted to sinus rhythm for these measurements. Ganglionic plexi isolation was tested by stimulation of the ganglionic zones around the pulmonary veins and at Waterston's groove at a rate of $1000 \mathrm{bpm}$ and an impulse amplitude of 18 volts (Oscor External pacemaker 203H; Oscor Inc, Palm Harbor, Fla) to check for sudden bradycardia, as described previously. ${ }^{8}$ When cardioversion was not successful, atrial electrical activity within the pulmonary veins (entrance block) and ganglionic plexi activity were measured.

Follow-up protocol included physical examination at the first, third, and sixth months after the procedure, 24-hour Holter monitoring between the fourth and sixth months and also at 1 year, and echocardiograms at 6 months. Success was defined as no symptomatic episodes of arrhythmia or no supraventricular arrhythmia lasting for more than 30 seconds in any electrocardiographic or Holter measurements. Preoperative antiarrhythmic therapy was maintained during follow-up in all patients postoperatively. After 3 months, antiarrhythmic drugs were suppressed except in patients with $\beta$-blocker therapy. Oral anticoagulation was maintained in the first 6 months to 1 year after the procedure and then withdrawn if there was no recurrence of $\mathrm{AF}$, assuming that both the physician and the patient were in agreement.

\section{Statistical Analysis}

Besides application of standard descriptive statistics, a predictive logistic regression model was built to calculate the probability of success or failure of the procedure with respect to several preoperative factors. We had the following strategy to select the variables that should be included in our model: First, univariate logistic regression was used to analyze the influence of all preoperative factors on outcome after the intervention. ${ }^{9}$ We selected for the multivariate analysis all the variables that produced estimations with a $P$ value $<.40$. Then, to select our final model we followed the strategy of analyzing all the possible resulting equations evaluating all combinations of the previously selected predictors and all first-degree interactions between them to evaluate confusion. All models were constructed according to the hierarchical principle. We evaluated 3 different adjustment measurements to select the best model: Mallows' Cp criteria adapted for logistic regression, ${ }^{9}$ the area under the curve (AUC), and the likelihood ratio. For the selected final model, both sensitivity and specificity were calculated as well as the $95 \%$ confidence interval (CI) for the AUC estimation using the receiver operating characteristic procedure.

All data are expressed as mean \pm standard deviation.

\section{RESULTS}

Before surgical ablation, measurements of pulmonary vein isolation showed that all patients had either one or both sides electrically connected to the heart, meaning that percutaneous ablation had been unsuccessful. After performing 3 ablation lines with the bipolar radiofrequency clamp, trying to reach as much atrial tissue as possible, we again tested for isolation. If isolation was not totally ensured, up to 5 new ablation lines were performed with the help of the bipolar clamp or the transpolar radiofrequency pen (Transpolar ablation pen, AtriCure). We ensured transmurality by exit block in all patients with paroxysmal and persistent $\mathrm{AF}$ and in 1 patient with long-standing persistent $\mathrm{AF}$. Entrance block was confirmed in the patients with longstanding persistent AF that could not be cardioverted. No ganglionic plexi stimulation was achieved after pulmonary vein isolation in any patient. In $28(87.5 \%)$ of 32 patients, isolation was complete after the first round ( 3 ablations). In 4 patients we needed to perform 5 ablations on one of the sides to complete isolation. Procedural times ranged from 3 to 5 hours.

Follow-up is $100 \%$ complete and included physical examination, Holter monitoring, and echocardiography. Mean follow-up was $16 \pm 11$ months (range, 6-45 months). There was no mortality during the procedure or follow-up (Table 2). Bilateral pulmonary vein isolation was completed in all except 2 patients with paroxysmal AF. In these patients, atrial bleeding required conversion to open right thoracotomy. One patient required extracorporeal circulation by femoral access to control hemorrhage, after bleeding from the IVC could not be controlled by hemostatic 4-0 polypropylene (Prolene; Ethicon, Inc, Somerville, NJ) stitches. Only right pulmonary vein isolation was performed in these patients and both remained in paroxysmal AF during followup. Atrial bleeding in both cases was due to an atrial lesion caused by cautery, attributed to our learning curve in this procedure. These patients were the only ones who required blood transfusion in the operating room, 2 and 4 units of

TABLE 2. Results

\begin{tabular}{ll}
\hline & \multicolumn{1}{c}{ Results } \\
\hline Peroperative or follow-up mortality & 0 \\
Hospital stay (d) & 6 (range, $4-15)$ \\
SPC & $32 / 34$ patients $(94 \%)$ \\
Need for extracorporeal circulation & $1(3 \%)$ \\
Free from AF at 6 mo (SPC) & $22 / 32$ patients $(69 \%)$ \\
Paroxysmal & $13 / 15$ patients $(87 \%)$ \\
Persistent & $8 / 12$ patients $(67 \%)$ \\
Long-standing persistent & $1 / 5$ patients $(20 \%)$ \\
Free from AF at 12 mo (SPC) & $16 / 26$ patients $(62 \%)$ \\
Paroxysmal & $9 / 11$ patients $(82 \%)$ \\
Persistent & $6 / 10$ patients $(60 \%)$ \\
Long-standing persistent & $1 / 5$ patients $(20 \%)$ \\
Off oral anticoagulation at 12 mo & $10 / 26$ patients $(63 \%)$ \\
\hline
\end{tabular}

$S P C$, Surgical procedure completed; $A F$, atrial fibrillation. 
blood, respectively. No bleeding was caused by repeated ablation although we limited application of bipolar radiofrequency to 5 times per side.

One patient, with persistent AF and a history of severe chronic obstructive pulmonary disease owing to smoking and 15 years of continuous amiodarone intake, required postoperative mechanical ventilatory support for 2 days. All other patients were extubated in the operating room. No other postoperative complications were registered in any patient. Mean hospital stay was 6 days (range, 4-15 days). The patient with prolonged ventilatory support and preoperative persistent $\mathrm{AF}$ was readmitted 4 weeks after surgical intervention because of sudden episodes of pain and dyspnea. Echocardiography and a 64-slice computed tomographic angiographic scan showed normal ejection fraction with no signs of pulmonary vein stenosis, pulmonary embolism, or cardiac tamponade. He was discharged home in better condition after 10 days and after the initiation of nonsteroidal anti-inflamatory therapy.

One patient with a history of persistent AF had a stroke 3 days after surgery. This patient had a residual superior left quadrantanopsia. Inasmuch as this patient had maintained sinus rhythm since surgery, with no anticoagulation or antiaggregation therapy, we changed our protocol to complete anticoagulation with intravenous sodium heparin 12 hours after the operation. The fourth patient on this protocol had a left chest wall hematoma and hemothorax 48 hours after the operation and 24 hours after removal of the chest tube, requiring blood transfusion ( 2 units of red cells), thoracoscopic drainage, and revision. This patient was the only one requiring blood transfusion in the postoperative period. Since then, our protocol includes exclusion of oral anticoagulation 2 days before surgery, administering aspirin 100 $\mathrm{mg}$, and restarting oral anticoagulation 24 hours after surgery. With this protocol, we have had no additional intraoperative or postoperative cases of bleeding or stroke.

Supraventricular arrhythmias has been eradicated in $82 \%$ of patients with preoperative paroxysmal AF at 1 year's follow-up, excluding the 2 patients in whom the procedure was not completed (9/11). Sixty percent of patients with a history of persistent $\mathrm{AF}$ have maintained sinus rhythm postoperatively (6/10). Only 1 of the 5 patients in the long-standing persistent AF group was able to maintain sinus rhythm postoperatively.

In patients considered to be without $\mathrm{AF}$ recurrence, sinus rhythm was recorded in all physical examinations after a 3month window after the operation; there was no trace of $\mathrm{AF}$ or flutter in any of the 24-hour Holter studies and patients reported no recurrence of their symptomatic arrhythmia, either palpitations or symptoms of heart failure. All patients were free of AF at 1 year $(62 \%, 16 / 26)$ and reported better quality of life in the postoperative visits. They reported that they were less anxious. They were successful at switching from oral anticoagulation to platelet antiaggregation, which could be achieved in 10 patients at 1 year after the operation. Nevertheless, the patient with quadrantanopsia continues to have several problems related to normal life activities, such as driving motor vehicles. Interestingly, some patients reported some of the same symptoms of discomfort that initiated AF before the operation, lasting for a few seconds at a time, but with no continuation to any supraventricular arrhythmia. Patients that remained in $\mathrm{AF}$ at 1 year reported no change in quality of life after the operation.

Four patients in the persistent AF group in whom AF continued for more than 3 months after surgery returned to the Electrophysiology Laboratory. Two of them had a change of pattern to left flutter, and 2 continued to have persistent AF. In all of them the pulmonary vein isolation performed during surgery was confirmed in the electrophysiology laboratory. In 1 patient with left atrial flutter, flutter was abolished and changed to sinus rhythm by performing a line between the right and left pulmonary veins, suggesting that the flutter pattern was generated by an electrical impulse going around one of the isolated areas. In the other 3 patients, no specific sources of arrhythmia were found within the multiple scars of the left atrium from prior ablations. Nevertheless, new lines were performed, with absence of late sinus rhythm.

As explained in the statistical part of the Methods section, a predictive logistic regression model was built to calculate the probability of success or failure of the procedure with respect to several preoperative factors. The univariate logistic regression analysis showed that left atrial diameter greater than $45 \mathrm{~mm}$ and AF type were the only statistically significant factors influencing success or failure (Table 3 ). Interestingly, the number of previous percutaneous ablations did not affect results. Neither gender nor age influenced the results.

As described in the Methods section and using the results from the univariate analysis, we selected the multivariate logistic regression model containing 3 variables that produced the best estimations of the adjustment criteria: left atrial size greater than $45 \mathrm{~mm}$, nonparoxysmal $\mathrm{AF}$, and more than 5 years in AF. Besides the statistical properties of the variables, we chose these variables because of their simplicity and because of the exactitude in their quantification. The calculated AUC using the receiver operating characteristic procedure for the model was 0.795 (95\% CI, 0.633-0.958; $P=.008$ ), which allows a sensitivity of $70 \%$ and specificity of $81.8 \%$. This model predicts an estimated risk of failure of surgical pulmonary vein isolation of $2.85 \%$ if no risk factor is present (95\% CI, 0.2-29.46). When all factors are present, the estimated risk of failure reaches $62 \%(95 \%$ CI, 33.8884.28) (Table 4). The relative risks of all possible patterns are shown in Table 5.

\section{DISCUSSION}

Our study corroborates prior knowledge that paroxysmal $\mathrm{AF}$ can be controlled by pulmonary vein isolation. For this to 
TABLE 3. Univariate logistic regression analysis. Odds Ratio (OR) for failure

\begin{tabular}{|c|c|c|c|c|c|}
\hline & & \multirow[b]{2}{*}{ OR } & \multicolumn{2}{|c|}{$95 \% \mathrm{CI}$} & \multirow[b]{2}{*}{$P$ value } \\
\hline & & & Low & High & \\
\hline $\begin{array}{l}\text { Left atrial } \\
\quad \text { size }(\mathrm{mm})\end{array}$ & $>45$ vs $\leq 45$ & 7.00 & 1.185 & 41.359 & .019 \\
\hline AF type & $\begin{array}{l}\text { All other } \\
\text { vs paroxysmal }\end{array}$ & 5.778 & 0.987 & 33.828 & .035 \\
\hline Gender & Male vs female & 1.583 & 0.0221 & 11.361 & .652 \\
\hline Duration of $\mathrm{AF}(\mathrm{y})$ & $\geq 5$ vs $<5$ & 5.143 & 0.547 & 48.365 & .102 \\
\hline Previous percutaneous procedures & $\begin{array}{l}\geq 2 \text { vs } 1 \\
>2 \text { vs } \leq 2\end{array}$ & $\begin{array}{l}1.333 \\
1.167\end{array}$ & $\begin{array}{l}0.267 \\
0.251\end{array}$ & $\begin{array}{l}6.653 \\
5.413\end{array}$ & $\begin{array}{l}.724 \\
.844\end{array}$ \\
\hline Age (y) & $\geq 60$ vs $<60$ & 2.267 & 0.453 & 11.349 & .322 \\
\hline
\end{tabular}

$C I$, confidence interval; $A F$, atrial fibrillation.

be effective, the ablation technique, either percutaneous or surgical, must ensure linearity and transmurality along the lesions. We performed a consistent ablation line that was documented to be transmural in the left atrium along the antrum of the pulmonary veins, eradicating paroxysmal AF. Inasmuch as our patients had multiple previous percutaneous radiofrequency ablations to isolate pulmonary veins, our results suggest that percutaneous ablation may not be totally effective at isolating pulmonary veins in some patients. The ineffectiveness of percutaneous ablation in patients with a left atrium larger than $45 \mathrm{~mm}$ may be due to long ablation lines, increasing the probability of leaving gaps along the dot-by-dot endocardial ablation lines. Also, possible failures may be due to increased myocardial thickness, which may, in turn, prevent endocardially applied monopolar radiofrequency from being transmural. In our opinion, our patients had incomplete vein isolation before surgery, and this was completed by bipolar radiofrequency. Our first conclusion is that failure of percutaneous ablation does not necessarily mean failure in the strategy therapy of pulmonary vein isolation in patients with paroxysmal AF.

Our results in patients with persistent $\mathrm{AF}$ are not so clear. Although a $60 \%$ success rate is not a failure in treatment, undoubtedly this shows that pulmonary isolation is not enough in a significant percentage of these patients. This may be due to other factors influencing the permanence of this arrhythmia. Our multivariate logistic regression model indicates that an effective pulmonary vein isolation, such as surgical

TABLE 4. Estimated risk of failure depending on predictive factors

\begin{tabular}{lc}
\hline \multicolumn{1}{c}{ Risk patterns } & Risk $(\mathbf{9 5} \% \mathbf{C I})$ \\
\hline No risk factors present & $2.85 \%(0.2-29.46)$ \\
Isolated risk factors & \\
$\quad$ Left atrial size $>45 \mathrm{~mm}$ & $9.42 \%(0.35-75.39)$ \\
$\quad$ Nonparoxysmal AF & $7.51 \%(0.35-65.34)$ \\
$>5$ y with AF & $14.46 \%(3.20-46.36)$ \\
All risk factors present & $62.37 \%(33.88-84.28)$ \\
\hline
\end{tabular}

$C I, 95 \%$ confidence interval for the estimation of the probability of failure of the procedure depending on the presence of preoperative risk factors predicted by our multivariate logistic regression model; $A F$, atrial fibrillation. bipolar radiofrequency is able to achieve, has between a $7 \%$ and a $15 \%$ chance of failure when $\mathrm{AF}$ is not paroxysmal, left atrial diameter reaches $45 \mathrm{~mm}$, or in patients with a history of AF lasting more than 5 years. Moreover, results are prohibitive when these 3 factors combine.

Most of the patients with persistent and long-standing persistent AF had a history of paroxysmal AF, so that the initial value of triggering zones around the pulmonary veins may have been an important factor in the past. However, the establishment of electrical re-entrant circuits in these enlarged and more fibrotic atria probably changes the atrial substrate, favoring the chronicity of AF and overriding the importance of the triggering factors that initially imposed AF. This is possibly why procedures designed to block re-entrant circuits, such as the Cox maze operation, are more successful in persistent and long-standing persistent AF. Previous reports of radiofrequency pulmonary vein isolation alone in patients with long-standing $\mathrm{AF}$ have reported between $25 \%$ and $72 \%$ success in recovering stable sinus rhythm. ${ }^{10,11}$ The $20 \%$ success rate in our subgroup of patients with repetitive failed catheter ablation further reinforces the idea that pulmonary vein isolation alone may be insufficient in long-standing persistent AF. Indication of percutaneous or minimally invasive ablation procedures solely involving pulmonary veins in this type of patient is controversial and needs to be in consensus with the patient, inasmuch as it involves trading minimal invasiveness for a lesser chance of success. Percutaneous endocardial

TABLE 5. Relative risk of all possible patterns

\begin{tabular}{lccc}
\hline & & \multicolumn{2}{c}{$\begin{array}{c}\text { Left atrial } \\
\text { size }(\mathbf{m m})\end{array}$} \\
\cline { 3 - 4 } Preoperative & Duration of & & \\
arrhythmia & $\mathbf{A F}(\mathbf{y})$ & $\leq \mathbf{4 5} \mathbf{~ m m ~ ( R R ) ~}$ & $>\mathbf{4 5} \mathbf{~ m m ~ ( R R ) ~}$ \\
\hline Paroxysmal & $<5 \mathrm{y}$ & 1.00 & 3.30 \\
& $\geq 5 \mathrm{y}$ & 5.07 & 13.15 \\
Other & $<5 \mathrm{y}$ & 2.63 & 7.84 \\
& $\geq 5 \mathrm{y}$ & 11.18 & 21.89 \\
\hline
\end{tabular}

$R R$, Relative risk of failure of all possible combinations of preoperative factors compared with the lowest risk model with no risk factors present; $A F$, atrial fibrillation. 
ablation techniques can further extend the ablation pattern by connecting lines to the mitral annulus or by isolating the posterior wall of the left atrium in what has been named the "box lesion." However, despite the latest technology, percutaneous ablation proves to be an aggressive therapy with a $50 \%$ success rate in moderately enlarged left atria. The incidence of major complications is $5 \% .^{4,6}$

Patients in this study represent our initial experience in thoracoscopic AF therapy. In our opinion, success rates regarding freedom from AF in this series were not affected by our reduced exposure to this operation. Not only does our group routinely use bipolar radiofrequency for AF treatment through sternotomy, but the device used ensures a transmural linear lesion of the tissue clamped, with minimal surgeon-induced variability. To further ensure transmurality, we performed tests of isolation and repeated the procedure up to 5 times on each side until isolation was confirmed. Results for maintenance of sinus rhythm were similar from the earlier to the later patients when matched for $\mathrm{AF}$ type. However, a learning curve had to be surpassed for surgeons not familiar with thoracoscopy. Operating times have ranged from 5 hours initially to 3 hours in the last 10 patients. Most important, we could not complete the ablation procedure in 2 patients owing to bleeding from the right atrium that became severe after we tried to repair it thoracoscopically. Urgent cardiopulmonary bypass through femoral access was required in 1 patient. Additionally, we had to change our anticoagulation and antiaggregation protocols after a patient with no antiaggregation or anticoagulation had a minor stroke, and he was followed by another patient with a hemothorax caused by an excess of anticoagulation emphasis on our part. We would like to bring a word of caution to surgeons entering thoracoscopic AF therapy. In an operation in which morbidity is not expected, 1 peroperative death has been reported. ${ }^{12}$ However, we believe thoracoscopic pulmonary vein isolation is a good alternative to percutaneous catheter ablation for paroxysmal AF, inasmuch as the results published for both techniques support minimally invasive surgery. The rates of major complications do not favor one technique over the other. ${ }^{4,5,7}$

\section{Study Limitations}

We acknowledge that this is a report with few patients, but in our opinion it indicates important differences in results of both catheter and thoracoscopic ablation. More series are needed to confirm this, preferably randomized studies comparing both techniques. A possible factor that needs to be considered with larger studies and clinical trials is the possibility of missing arrhythmias during follow-up, including the use of transtelephonic monitoring, which of course may not always be available, to rule out asymptomatic AF recurrences. Patients in this study are a highly selected group in whom catheter ablation failed, with a high incidence of major complications. Nevertheless, we wish to highlight that this is a small population from an experienced electrophysiology group with more than $100 \mathrm{AF}$ ablation procedures per year and published results. ${ }^{7}$

We conclude that minimally invasive pulmonary vein isolation is successful in controlling paroxysmal AF no matter whether previous multiple unsuccessful catheter ablations have been performed. The cause of percutaneous catheter ablation failure may be due to a lack of pulmonary vein isolation. On the other hand, thoracoscopy-assisted pulmonary vein isolation did not provide efficient control of persistent $\mathrm{AF}$ and failed to control long-standing persistent $\mathrm{AF}$, probably owing to an alteration in the atrial substrate that overrides the importance of the triggering zones around pulmonary veins. Last, the technical procedure was feasible and reproducible, although as with other minimally invasive surgical operations, there is a learning curve that needs to be confronted.

\section{References}

1. Fuster V, Ryden LE, Cannom DS, Crijns HJ, Curtis AB, Ellenbogen KA, et al. [ACC/AHA/ESC 2006 guidelines for the management of patients with atrial fibrillation-excutive summary] [in Portuguese]. Rev Port Cardiol. 2007;26: 383-446.

2. Haissaguerre M, Jais P, Shah DC, Takahashi A, Hocini M, Quiniou G, et al. Spontaneous initiation of atrial fibrillation by ectopic beats originating in the pulmonary veins. $N$ Engl J Med. 1998;339:659-66.

3. Calkins H, Brugada J, Packer DL, Cappato R, Chen SA, Crijns HJ, et al. HRS/ EHRA/ECAS expert consensus statement on catheter and surgical ablation of atrial fibrillation: recommendations for personnel, policy, procedures and follow-up. A report of the Heart Rhythm Society (HRS) Task Force on Catheter and Surgical Ablation of Atrial Fibrillation developed in partnership with the European Heart Rhythm Association (EHRA) and the European Cardiac Arrhythmia Society (ECAS); in collaboration with the American College of Cardiology (ACC), American Heart Association (AHA), and the Society of Thoracic Surgeons (STS). Endorsed and approved by the governing bodies of the American College of Cardiology, the American Heart Association, the European Cardiac Arrhythmia Society, the European Heart Rhythm Association, the Society of Thoracic Surgeons, and the Heart Rhythm Society. Europace. 2007;9:335-79.

4. Cappato R, Calkins H, Chen SA, Davies W, Iesaka Y, Kalman J, et al. Worldwide survey on the methods, efficacy, and safety of catheter ablation for human atrial fibrillation. Circulation. 2005;111:1100-5.

5. Wolf RK, Schneeberger EW, Osterday R, Miller D, Merrill W, Flege JB Jr, et al. Video-assisted bilateral pulmonary vein isolation and left atrial appendage exclusion for atrial fibrillation. J Thorac Cardiovasc Surg. 2005;130:797-802.

6. Oral H, Knight BP, Ozaydin M, Chugh A, Lai SW, Scharf C, et al. Segmental ostial ablation to isolate the pulmonary veins during atrial fibrillation: feasibility and mechanistic insights. Circulation. 2002;106:1256-62.

7. Berruezo A, Tamborero D, Mont L, Benito B, Tolosana JM, Sitges M, et al. Pre-procedural predictors of atrial fibrillation recurrence after circumferential pulmonary vein ablation. Eur Heart J. 2007;28:836-41.

8. Mehall JR, Kohut RM Jr, Schneeberger EW, Taketani T, Merrill WH, Wolf RK. Intraoperative epicardial electrophysiologic mapping and isolation of autonomic ganglionic plexi. Ann Thorac Surg. 2007;83:538-41.

9. Hosmer DW, Hosmer T, Le CS, Lemeshow S. A comparison of goodness-of-fit tests for the logistic regression model. Stat Med. 1997;16:965-80.

10. Gaita F, Riccardi R, Caponi D, Shah D, Garberoglio L, Vivalda L, et al. Linear cryoablation of the left atrium versus pulmonary vein cryoisolation in patients with permanent atrial fibrillation and valvular heart disease: correlation of electroanatomic mapping and long-term clinical results. Circulation. 2005;111:136-42.

11. Sagbas E, Akpinar B, Sanisoglu I, Caynak B, Tamtekin B, Oral K, et al. Videoassisted bilateral epicardial pulmonary vein isolation for the treatment of lone atrial fibrillation. Ann Thorac Surg. 2007;83:1724-30.

12. Edgerton JR, Edgerton ZJ, Weaver T, Reed K, Prince S, Herbert MA, et al. Minimally invasive pulmonary vein isolation and partial autonomic denervation for surgical treatment of atrial fibrillation. Ann Thorac Surg. 2008;86:35-8. 